\title{
A lower bound for the velocity of quantum communications in the preferred frame.
}

\author{
B. Cocciaro ${ }^{\mathrm{a}, *}$, S. Faetti ${ }^{\mathrm{b}}$, L. Fronzonic ${ }^{\mathrm{c}}$ \\ ${ }^{a}$ Liceo Scientifico XXV Aprile, via Milano 2, 56025 Pontedera (Pi) Italy \\ ${ }^{b}$ Dipartimento di Fisica and Polylab of INFM, Largo B. Pontecorvo, 56123 Pisa, Italy \\ ${ }^{c}$ Dipartimento di Fisica and INFMCRS-Soft, Largo B. Pontecorvo, 56123 Pisa, Italy
}

\begin{abstract}
An EPR experiment with polarized entangled photons is performed to test the Eberhard model. According to the Eberhard model, quantum correlations between space-like separated events are due to a superluminal communication signal propagating in a preferred frame. The coincidences between entangled photons passing through two polarizers aligned along a East-West axis are measured as a function of time during 21 sidereal days. No deviation from the predictions of the Quantum Theory is observed. Tacking into account for the experimental uncertainties, we infer that, if a preferred frame for superluminal signals exists which moves at velocity $\vec{v}$ with respect to the Earth, the modulus of the velocity of quantum communications in this frame has to be greater than $v_{t} \simeq 0.6 \cdot 10^{4} c$ for $v<0.1 c$ and for any arbitrary direction of $\vec{v}$.
\end{abstract}

Keywords: Optical EPR experiments, Preferred frame, Tachyons

\section{Introduction}

The non-local character of Quantum Mechanics $(Q M)$ has been object of a great debate starting from the famous Einstein-Podolsky-Rosen $(E P R)$ paper [1]. Consider, for instance, a quantum system made by two photons 1 and 2 that are in the polarization entangled state

$$
\mid A>=\frac{1}{\sqrt{2}}\left(\left|H, H>+e^{i \phi}\right| V, V>\right)
$$

where $H$ and $V$ stand for horizontal and vertical polarization, respectively, and $\phi$ is a constant phase coefficient. The two photons propagate in space far away one from the other and the polarization of the two photons is measured at a given time. According to $Q M$, a measurement of horizontal polarization of one of the entangled photons leads to the collapse of the entangled state to $|H, H\rangle$, then, also the other photon must collapse to the horizontal polarization whatever is its distance from the previous

\footnotetext{
* Corresponding author

Email addresses: b.cocciaro@comeg.it (B. Cocciaro), faetti@df.unipi.it (S. Faetti), fronzoni@df.unipi.it (L. Fronzoni)
}

photon. This behavior suggests the existence of a sort of "action at distance" qualitatively similar to that between electric charges and masses that was introduced before the advent of the local Maxwell electromagnetic theory and the local Einstein General Relativity theory. Many physicists are unsatisfied of the non-local character of $Q M$ and alternative local models based on hidden variables have been suggested. As shown by Bell [2] and other authors [3, 4], local hidden variables theories must satisfy some inequalities that are not satisfied by $Q M$. Many experiments have been performed for checking these inequalities $[5-13]$ and the validity of $Q M$ versus local hidden variables theories has been always found. Although the locality loophole and the detection loophole have not yet completely closed using a single experimental apparatus [14], experiments have continuously converged to closing both the locality loophole [6, 7, 10, 11] and the detection loophole [8, 12]. Then, it seems to us to be reasonable to think that hidden variables alone cannot fully justify $Q M$ correlations of entangled particles. In this case, alternative local explanations of $Q M$ correlations could be possible assuming some communication between entangled particles [15, 16]. 
Indeed, as shown by Eberhard [15], a realistic local model of $Q M$ can be obtained assuming that some superluminal communication (quantum tachyon) propagating in a preferred frame $(P F)$ can occur in a quantum system. Tachyons are known to lead to causal paradoxes 17] (the present in a given point can be affected by the future in the same point), but it can be shown that no causal paradox arises if they propagate in a preferred frame where the tachyon velocity $v_{t}=\beta_{t} c\left(\beta_{t}>1\right)$ is the same in all directions (see, for instance, section 3.1 of [18]). According the Eberhard model, if two photons are in the entangled state of eq.(1), when polarization of one photon is measured and it collapses in the horizontally oriented state, then a tachyon is sent to the other entangled photon that collapses to the horizontally oriented state only after this communication has been received. Therefore, the standard quantum correlations for the polarization measurements of entangled photons can be recovered only if it has been communication between the two photons. If there has been not sufficient time for this communication, a lack of quantum correlation occurs. Of course, the predictions of the Eberhard model coincide entirely with those of $Q M$ if $v_{t} \rightarrow \infty$, then no experiment satisfying $Q M$ can invalidate this model but it can only fix a lower bound for the tachyon velocity $v_{t}$.

A long-distance $(10.6 \mathrm{~km})$ EPR experiment has been performed by Scarani et al. [19] using energytime entangled photons to establish a lower bound for velocity $v_{t}$ of quantum communications. The experimental results were analyzed under the assumption that the $P F$ is the frame of cosmic microwave background radiation. With this assumption, they obtained a lower bound $v_{\min }=1.5 \cdot 10^{4} \mathrm{c}$. More recently similar measurements have been performed by Salart et al. 20] improving some features of the previous experiment and using detectors aligned close to East-West direction (at angle $\alpha=5.8^{\circ}$ ). In such a way the authors were able to find a higher value of the lower bound of the tachyon velocity for any direction of velocity $\vec{v}$ of the $P F$. In this Letter we report the results of a small distance $(2 \mathrm{~m})$ $E P R$ experiment with polarization entangled photons. The polarization measurements on the two entangled photons are made in two points $A$ and $B$ aligned along the East-West direction. Whatever is the orientation of velocity $\vec{v}$ of the preferred frame, an appreciable disagreement with quantum predictions would be expected if the tachyon velocity in the $P F$ is lower than a minimum value $\beta_{t, \min } c$ (see eq.(12)). Since we do not find any deviation from the predictions of $Q M$, we infer that possible tachyons velocity exceed $\beta_{t, \min } c$ in agreement with the results in refs. [19] and [20]. In Section 2 we discuss what are the conditions that make possible the experimental observation of a lack of quantum correlation. In Section 3 we discuss the main features of our apparatus and the main experimental uncertainties. In Section 4 we report our results and the conclusions.

\section{Conditions for the lack of quantum corre- lation of entangled photons.}

The main features of the experimental method are schematically drown in fig, 1, Two entangled photons are generated at a point $P$ and meet two polarizers at points $A$ and $B$ at distance $d_{A B}=$ $x_{B}-x_{A}>0$ along a $x$-axis of the laboratory frame oriented along the East-West direction.

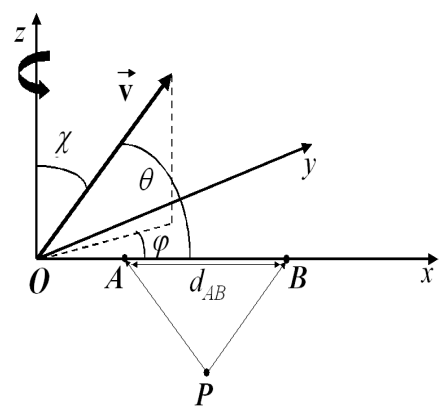

Figure 1: $P$ : position of the source of the entangled photons; $A, B$ : positions of the polarizers; $\vec{v}$ : velocity of the tachyons preferred frame $(P F) ; x$ : East-West axis in the laboratory; $z$ : rotation axis of the Earth.

The $z$-axis is parallel to the rotation axis of the Earth. We assume that a tachyon going from $A$ to $B$ or from $B$ to $A$ is emitted as soon as a photon reaches point $A$ or $B$, respectively. At a given time $t$, velocity $\vec{v}(v=\beta c, 0 \leq \beta<1)$ of the tachyon $P F$ with respect to the laboratory frame makes the polar angle $\chi$ with the $z$-axis and the azimuthal angle $\varphi$ with the $x$-axis. Due to the Earth motion, angle $\varphi$ changes according to $\varphi(t)=\frac{2 \pi}{T} t+\varphi_{0}$, where $T$ is the sidereal day. Using the Lorentz transformations with $\beta_{t}>1$ we find that velocity $v_{t}^{+}(\theta)$ of a tachyon going from $A$ to $B$ in the laboratory frame 
is:

$$
v_{t}^{+}(\theta)=c \frac{\sqrt{\left[1+\beta \beta_{t} C(\theta)\right]^{2}+\left(\beta_{t}^{2}-1\right)\left(1-\beta^{2}\right)}}{1+\beta \beta_{t} C(\theta)}
$$

where

$$
\begin{gathered}
C(\theta)=\left[\beta_{t}\left(1-\beta^{2} \cos ^{2} \theta\right)\right]^{-1}\left[-\beta \sin ^{2} \theta+\right. \\
\left.\cos \theta \sqrt{1-\beta^{2}} \sqrt{\left(\beta_{t}^{2}-1\right)\left(1-\beta^{2} \cos ^{2} \theta\right)+1-\beta^{2}}\right] .
\end{gathered}
$$

Then, velocity $v_{t}^{-}(\theta)$ of a tachyon that propagates in the opposite direction of the $x$-axis (from $B$ to $A$ ) at angle $\pi-\theta$ with respect to velocity $\vec{v}$ is

$$
v_{t}^{-}(\theta)=v_{t}^{+}(\pi-\theta) .
$$

We emphasize that velocities $v_{t}^{+}(\theta)$ and $v_{t}^{-}(\theta)$ given in eqs. (2) and (4) can assume negative values as it is evident in fig. 22 where $c / v_{t}^{+}(\theta)$ and $-c / v_{t}^{-}(\theta)$ are drawn. It is important to remark that $v_{t}^{+}(\theta)$ represents the velocity of a tachyon going from $A$ to $B$ toward the positive direction of the $x$-axis. Since $v_{t}^{+}(\theta)=\frac{x_{B}-x_{A}}{t_{B}-t_{A}}$ and $x_{B}-x_{A}>0$, a negative value of $v_{t}^{+}(\theta)$ simply means that $t_{B}<t_{A}$, that is a tachyon emitted at $A$ when the clock fixed in $A$ signs $t_{A}$ meets point $B$ when the clock fixed in $B$ signs $t_{B}<t_{A}$. This is not surprising if one takes into account that clocks at $A$ and $B$ are synchronized using the standard Einstein synchronization procedure and, thus, times $t_{A}$ and $t_{B}$ measured in different space points have not an absolute meaning. For a more detailed discussion of this point we refer to 21$]$.

We denote by $\Delta$ the optical path difference of entangled photons $\left(t_{B}=t_{A}+\Delta / c\right)$. Quantum correlations between the polarizations of the entangled photons at points $A$ and $B$ will be recovered if one of these conditions is satisfied:

a) a tachyon emitted at $A$ at time $t_{A}$ meets $B$ at time $t_{B}^{\prime}=t_{A}+d_{A B} / v_{t}^{+}(\theta)<t_{B}$ (before the arrival of the other entangled photon at $B$ );

b) a tachyon emitted at $B$ at time $t_{B}$ meets $A$ at time $t_{A}^{\prime}=t_{B}+d_{A B} / v_{t}^{-}(\theta)<t_{A}$ (before the arrival of the other entangled photon at $\mathrm{A}$ ).

Quantum correlations will be not observed if none of these conditions is satisfied, that is if:

$$
t_{A}+\frac{d_{A B}}{v_{t}^{+}(\theta)}>t_{A}+\frac{\Delta}{c} \quad \vee \quad t_{B}+\frac{d_{A B}}{v_{t}^{-}(\theta)}<t_{B}-\frac{\Delta}{c} .
$$

We remark that our theoretical analysis is made under the well-founded assumption that the polarization measurement takes place inside the polarizing layers. From conditions (5) we get:

$$
-\frac{c}{v_{t}^{-}(\theta)}<\rho<\frac{c}{v_{t}^{+}(\theta)} \text {. }
$$

where $\rho=\Delta / d_{A B}$.

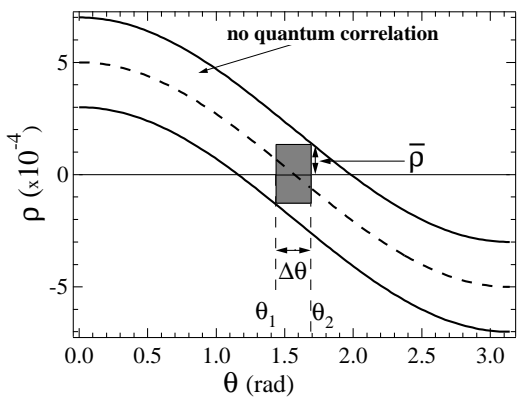

Figure 2: The full curves represent functions $-c / v_{t}^{-}(\theta)$ (lower curve) and $c / v_{t}^{+}(\theta)$ (upper curve), respectively, for $\beta=0.5$. $10^{-3}$ and $\beta_{t}=0.5 \cdot 10^{4}$. The broken curve represents function $y=\beta \cos \theta$. The region between the full curves corresponds to the region where no quantum correlation occurs (eq. 6]).

Fig 2 shows an example of the $\theta$-dependence of functions $-c / v_{t}^{-}(\theta)$ (lower full curve) and $c / v_{t}^{+}(\theta)$ (upper full curve) together with function $y=\beta \cos \theta$ (broken middle curve) for $\beta=0.5 \cdot 10^{-3}$ and $\beta_{t}=0.5 \cdot 10^{4}$. For any value of angle $\theta$, there is always a finite interval of values of $\rho$ (above and below $\beta \cos \theta$ ) that satisfies condition (6). Using eqs. (2)-(5), it can be shown that this property remains satisfied for any $\beta<1$ and $\beta_{t}>1$. For instance, in the case shown in fig 2, no quantum correlation occurs in a finite interval of $\theta$-angles if $-7 \cdot 10^{-4}<$ $\rho<7 \cdot 10^{-4}$. At the first order in $1 / \beta_{t}$ inequality (6) reduces to $\beta \cos \theta-a(\beta, \theta) / \beta_{t}<\rho<\beta \cos \theta+a(\beta, \theta) / \beta_{t}$, where $a(\beta, \theta)=\sqrt{1-\beta^{2}} \sqrt{1-\beta^{2} \cos ^{2} \theta}$. In our experiment, angle $\theta$ between velocity $\vec{v}$ of the $P F$ and the $x$-axis (East-West direction) will change periodically due to the Earth rotation. In particular

$$
\theta(t)=\arccos [-\sin \chi \sin \omega t]
$$

where we assumed $\theta=\pi / 2$ at $t=0$ and where $\omega$ is the angular velocity of the Earth and $t$ is the time. Angle $\theta$ will oscillate periodically between a minimum value $\pi / 2-\chi(\varphi(t)=0$ in fig 1) and a maximum value $\pi / 2+\chi(\varphi(t)=\pi$ in fig (1). Looking at fig, 2 we see that, for $\Delta=0$, there exists always a finite interval of angles where no quantum 
correlation must occur. Then, it is convenient to set $\Delta=0$ in the experiment. However, the presence of an experimental uncertainty $\delta \Delta$ sets severe limitations to the possibility of observing a lack of quantum correlation.

In our experiment, distance $d_{A B}$ between the polarizer layers at $A$ and $B$ is $d_{A B}=1.75 \mathrm{~m}$ and the main experimental uncertainties are:

1) the uncertainty on the positioning of polarizers at the same distance from the source of entangled photons $(\delta \Delta \approx 40 \mu m)$;

2) the intrinsic uncertainty related to the coherence length $h_{c}=\lambda^{2} / \Delta \lambda$ of the entangled photons. The entangled photons pass through a $40 \mathrm{~nm}$ width optical filter centered at $820 \mathrm{~nm}$ leading to $h_{c} \approx 16 \mu \mathrm{m}$; 3 ) the spatial width of the active polarizing layers that is $d_{c} \approx 220 \mu \mathrm{m}$.

The resulting uncertainty is $\delta \Delta \approx 280 \mu \mathrm{m}$, then, in our experiment

$$
\rho=0 \pm \bar{\rho}= \pm 1.6 \cdot 10^{-4}
$$

where

$$
\bar{\rho}=\frac{\delta \Delta}{d_{A B}} .
$$

A total lack of quantum correlation will be only observed if angle $\theta$ is within the interval $\left[\theta_{1}, \theta_{2}\right]$ of amplitude $\Delta \theta$ shown in fig 2. Since $\theta=\theta(t)$, the condition $\theta_{1}<\theta<\theta_{2}$ establishes a corresponding time interval $\Delta t\left[\theta(\Delta t / 2)-\theta(-\Delta t / 2)=\theta_{2}-\theta_{1}\right]$. Then,

$$
\cos \theta_{1,2}=\cos \left[\theta\left(\mp \frac{\Delta t}{2}\right)\right]=-\sin \chi \sin \left(\mp \frac{\omega \Delta t}{2}\right),
$$

where symbols - and + refer to $\theta_{1}$ and $\theta_{2}$, respectively. The maximum value of interval $\Delta \theta$ for which a total lack of correlation can be observed is fixed by $\bar{\rho}$ and will be obtained using the condition

$$
2 \bar{\rho}<\frac{c}{v_{t}^{+}\left(\theta_{2}\right)}+\frac{c}{v_{t}^{-}\left(\theta_{1}\right)}
$$

that corresponds to impose that the rectangle of sides $\Delta \theta$ and $2 \bar{\rho}$ is fully inside the region limited by the curves $c / v_{t}^{+}(\theta)$ and $-c / v_{t}^{-}(\theta)$ (see the gray rectangle in fig(2). Substituting the expressions of $v_{t}^{+}(\theta)$ and $v_{t}^{-}(\theta)$ given in eqs. (2)-(4) into (11) with $\cos \theta_{1}$ and $\cos \theta_{2}$ given by (10), after some tedious but straightforward calculations we finally obtain:

$$
\beta_{t}<\beta_{t, \min }=\sqrt{1+\frac{\left(1-\beta^{2}\right)\left[1-\bar{\rho}^{2}\right]}{\left[\bar{\rho}+\beta \sin \chi \sin \frac{\omega \Delta t}{2}\right]^{2}}} .
$$

Equation (12) was already obtained by Salart et al.[20] and establishes the basic dependence of the maximum detectable tachyon velocity $\beta_{t, \min }$ on the experimental parameters $\bar{\rho}=\delta \Delta / d_{A B}$ and $\Delta t$. For a fixed value of $\beta, \beta_{t, \min }$ decreases increasing both $\bar{\rho}$ and $\Delta t(\Delta t<\pi / \omega)$.

In conclusion, in our experiment, whatever is velocity $\vec{v}$ (modulus $\beta c$ and direction $\chi$ ) of the $P F$, deviations from the predictions of the Quantum Mechanics should be always observed provided the tachyon velocity in the $P F$ satisfies condition (12).

\section{3. - Experimental apparatus and proce- dures.}

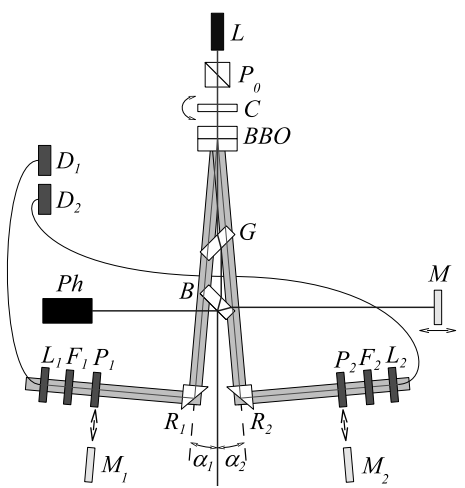

Figure 3: schematic view of the experimental apparatus

The experimental apparatus is schematically drawn in fig. A. A blue diode laser beam $(L, \lambda=$ $407 \mathrm{~nm}$ ) is polarized at $45^{\circ}$ with respect to the vertical axis by polarizer $P_{0}$, passes through a tilting plate compensator $(C)$ with vertical extraordinary axis and impinges at normal incidence on two thin $(0.5 \mathrm{~mm})$ adjacent nonlinear optical crystals $(B B O)$ cut for type-I phase matching. The two crystals are aligned so that their optic axes lie in planes perpendicular to each other with the first plane that is horizontal. The coherence length of the blue laser beam was increased to $1 \mathrm{~mm}$ using a reflection diffraction grating that is not shown in fig.3. Since the pump polarization is set at $45^{\circ}$, it induces down conversion at $\lambda=814 \mathrm{~nm}$ in each crystal [22]. The output photons are created in the maximally entangled state $\left(\left|H, H>+e^{i \phi}\right| V, V>\right) / \sqrt{2}$, where phase $\phi$ can be adjusted via tilting of the optical compensator $C$. The remaining components $\left(R_{i}, P_{i}, F_{i}, L_{i}\right.$ and $D_{i}$ ) are mounted on two $L$-shaped guides that can rotate in the horizontal plane of the optical table around a vertical axis passing for the center of 
the $B B O$ plates so that angles $\alpha_{1}$ and $\alpha_{2}$ can be changed continuously with an accuracy of $0.02^{\circ} . R_{1}$ and $R_{2}$ are two right angle prisms, $P_{1}$ and $P_{2}$ are thin polarizing films (LPNIR, Thorlabs), $F_{1}$ and $F_{2}$ are interference filters $(\lambda=820 \mathrm{~nm} \pm 20 \mathrm{~nm})$ and $D_{1}$ and $D_{2}$ are single photons counters (Perkin Elmer SPCM-AQ4C). $L_{1}$ and $L_{2}$ are optical lenses that focus the entangled photons on two multimode fibers connected to detectors $D_{1}$ and $D_{2}$. The detectors outputs are sent to electronic counters and to a coincidence circuit and, after, to a $P C$. A labview program controls any experimental feature. The $L$-shaped guides are rotated to maximize the count rates and the coincidences of the entangled photons. This condition is satisfied for $\alpha_{1}=\alpha_{2}=2^{\circ}$. In this condition, the centers of polarizers $P_{1}$ and $P_{2}$ are aligned along a $x$-axis in the East-West direction within $0.2^{\circ}$. To minimize any possible thermal displacement, the room temperature is held fixed at $27^{\circ} \pm 0.3^{\circ} \mathrm{C}$ during the whole measurements time.

In fig 3 are also shown a glass plate $G$, a beam splitter $B$, a reference mirror $M$ and a photodiode $P h$. These components are introduced only during the preliminary measurements to equalize the optical paths 1 and 2 of entangled photons from the $B B O$ plates to the first surfaces of polarizers $P_{1}$ and $P_{2}$ using an interferometric method. Glass plate $G$ is introduced to compensate the lateral shift of the blue laser beam due to beam splitter $B$. $G$ and $B$ are mounted in such a way that the rotation of the $L$-shaped guides is not disturbed. The first step consists on replacing polarizer $P_{1}$ by a mirror $M_{1}$, then the left $L$-shaped guide is rotated up to $\alpha_{1}=0$. In this condition, paths $B-R_{1}-M_{1}$ and $B-M$, constitute the two arms of a Michelson-like interferometer for the blue laser beam. Then, interference fringes occur in front of photodiode $P h$. Due to the finite coherence length of our blue laser beam (about $1 \mathrm{~mm}$ ), the maximum contrast of interference fringes is obtained for equal lengths of the two arms of the interferometer. To measure the fringe contrast, we put a small loudspeaker oscillating at a frequency of $100 \mathrm{~Hz}$ in the contact with the reference arm of the interferometer containing mirror $M$. This procedure induces small changes $(\lesssim 1 \mu \mathrm{m})$ of the length of the reference arm and, thus, a corresponding oscillation of the pattern of interference fringes in front of photodiode $P h$. Then, the amplitude of the oscillating signal at the output of photodiode $P h$ is proportional to the fringe contrast. The reference mirror $M$ is mounted on a motorized linear stage and the mirror position leading to the maximum amplitude of the oscillating signal is found with an estimated precision better than $5 \mu \mathrm{m}$. The same kind of measurement is, then, repeated with the right $L$-shaped guide after polarizer $P_{2}$ has been replaced by mirror $M_{2}$. In this latter case, the motorized linear stage of the reference mirror $M$ is held at the rest while $M_{2}$ is translated up to recover the maximum contrast of interference fringes. In these conditions the optical paths of entangled photons 1 and 2 (up to mirrors $M_{1}$ and $M_{2}$ ) are the same within $10 \mu \mathrm{m}$. Due to thermal effects, small changes of the lengths of the two arms could occur. To take under control this possible effect, we have repeated the measurement during a week at different times measuring the variations of differences of lengths of the interferometer arms. These variations are usually smaller than $5 \mu \mathrm{m}$ but, in a few cases, changes up to $25 \mu \mathrm{m}$ have been observed. The final procedure consists on replacing mirrors $M_{1}$ and $M_{2}$ with polarizers $P_{1}$ and $P_{2}$ in such a way that the first surface of the polarizing films (firstly encountered by the entangled photons) lies in the same position of the mirror surfaces. This is obtained using a profilometer that ensures a final accuracy better than $5 \mu \mathrm{m}$. Therefore, tacking into account for possible thermal drifts, the resulting difference of optical paths between the entangled photons in the two arms is estimated to be less than $40 \mu \mathrm{m}$.

Once the two optical paths have been equalized, the components $G, B, M$ and $P h$ are removed and plate $G$ is replaced by a black light absorber, then the $L$-shaped guides are positioned at angles $\alpha_{1}=\alpha_{2}=2^{\circ}$ where the maximum of coincidences is observed. We denotes here by $\gamma_{1}$ and $\gamma_{2}$ the angles between the polarization axes of polarizers $P_{1}$ and $P_{2}$ and the vertical axis. According to $Q M$, the probability of coincident detection of photons passing through the polarizers is

$$
P_{12}\left(\gamma_{1}, \gamma_{2}\right)=\frac{\left|\cos \gamma_{1} \cos \gamma_{2}+e^{i \phi} \sin \gamma_{1} \sin \gamma_{2}\right|^{2}}{2}
$$

whilst the probability of detection of a photon passing through a single polarizer is

$$
P_{1}\left(\gamma_{1}\right)=P_{2}\left(\gamma_{2}\right)=\frac{1}{2}
$$

In our experiment we set $\gamma_{1}=\gamma_{2}=\pi / 4$ and, thus, from eq. (13) we infer that the number of coincidences depends on phase $\phi$ according to 


$$
n_{\text {coinc }}(\phi)=n_{\max } \cos ^{2} \frac{\phi}{2} .
$$

The result in eq. (15) is predicted by $Q M$ and by the Eberhard model if there has been sufficient time for communication between photons 1 and 2 . On the contrary, if communication is not possible (see section 2), the Eberhard model predicts that the measurements at points $A$ and $B$ are fully uncorrelated (eqs. (4.68) and (4.81) in [15]) and the probability of coincident detection of photons becomes $P_{12}\left(\gamma_{1}, \gamma_{2}\right)=P_{1}\left(\gamma_{1}\right) P_{2}\left(\gamma_{2}\right)=1 / 4$ that leads to the constant coincidences rate

$$
n_{\text {coinc }}^{*}=\frac{n_{\max }}{2} .
$$

If $\phi=\pi$, the $Q M$ coincidence rate predicted by eq. (15) becomes $n_{\text {coinc }}=0$. Then, according to the Eberhard model, a sharp variation from the $Q M$ value $n_{\text {coinc }}=0$ to the uncorrelated one $n_{\text {coinc }}^{*}=$ $n_{\max } / 2$ should be observed in a suitable time interval of a sidereal day if $\phi=\pi$ and $\beta_{t}<\beta_{\text {min }}$ given in eq. (12).

\section{4. - Experimental results and conclusions.}

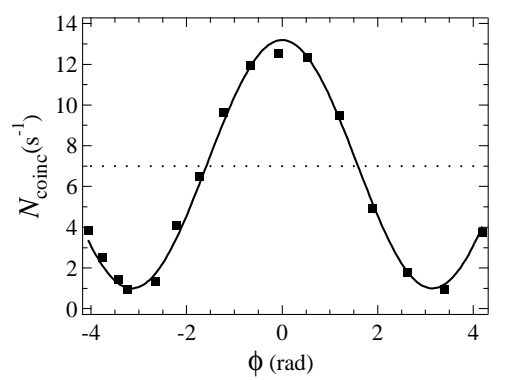

Figure 4: Coincidence rate (counts/s) versus the average phase $\phi$. The acquisition time is $\Delta t=100 \mathrm{~s}$. The full line is the best fit with function $N_{\text {coinc }}=a+b \cos ^{2}\left(\frac{\phi}{2}\right)$ with $a=1.14 s^{-1}$ and $b=12.05 s^{-1}$. The dotted horizontal line represents the theoretical prediction of the Eberhard model if quantum communication are not allowed (eq. (16)).

Fig 4 shows the experimental values of the coincidence rate $\left(N_{\text {coinc }}=n_{\text {coinc }} / \Delta t\right)$ versus phase $\phi$ that are obtained using a $100 s$ acquisition time and setting the polarizers angles at $\gamma_{1}=\gamma_{2}=\pi / 4$. The full line represents the best fit with function $N_{\text {coinc }}=a+b \cos ^{2}(\phi / 2)$ that differs from the theoretical prediction in eq. (15) due to the presence of constant $a$. The dotted horizontal line represents the theoretical prediction for no quantum communication. The non-vanishing value of coefficient $a$ is due in part to the dark spurious coincidences $\left(N_{\text {dark }}=0.3 s^{-1}\right)$ and in part $\left(0.84 s^{-1}\right)$ to the finite acceptance angle of entangled photons that is estimated to be $\Delta \alpha \approx 5 \cdot 10^{-3} \mathrm{rad}$ in our experiment. In fact, due to the birefringence of $B B O$, the phases differences between entangled photons that are emitted at slightly different angles have not the same values leading to the reduced contrast observed in fig 4 [23.

In order to detect a possible lack of quantum correlation occurring at a given time interval during a sidereal day, we set $\gamma_{1}=\gamma_{2}=\pi / 4$ and $\phi=\pi$. Indeed, according to eqs. (15) and (16), the maximum departure between quantum correlated and uncorrelated results (full and dotted lines in fig 4) is expected if phase $\phi$ is a multiple of $\pi$. We choose an odd multiple of $\pi$ since it corresponds to a minimum of the coincidence number $n_{\text {coinc }}$ and, thus, to a minimum value of the statistical noise $\sqrt{n_{\text {coinc }}}$. According to eq. (12), the higher value of the lower limit $\beta_{\text {min }}$ would be obtained for $\Delta t \rightarrow 0$. However, a reduction of the acquisition time leads to a decrease of the signal to noise ratio. In our experiment, sufficient signal to noise ratio is obtained setting $\Delta t=4 \mathrm{~s}$. With this choice, $\beta \sin \chi \sin \frac{\omega \Delta t}{2}<\bar{\rho}$ for any value of $\beta$ and $\chi$ and, thus, the finite acquisition time affects appreciably $\beta_{\text {min }}$ only for relativistic values of $\beta$. Using this acquisition time, we measure the number of coincidences at different times during a sidereal day. The

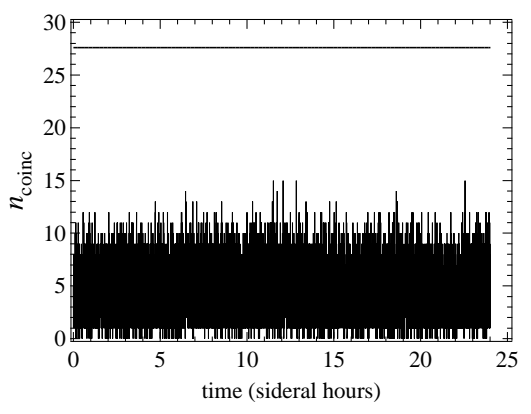

Figure 5: Number of coincidences versus time expressed in sidereal hours. The acquisition time is $\Delta t=4 \mathrm{~s}$. The full line is the number of coincidences predicted for no quantum correlation between entangled photons.

results of this measurement are shown in fig 5 where the horizontal line represents the value of coincidences expected for totally uncorrelated photons $\left[n_{\text {coinc }}=(a+b / 2) \Delta t\right]$. Although the experimen- 


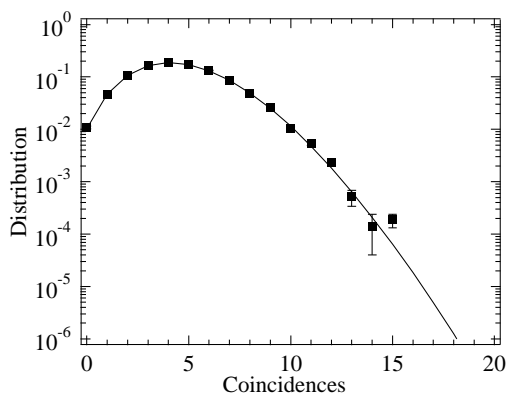

Figure 6: Relative frequency distribution of coincidences in a simple sidereal day for a $4 s$ acquisition time. The experimental data are those of fig 5 The full line represents the theoretical Poisson distribution.

tal noise is somewhat high due to the small acquisition time, the coincidences rate remains always well below the horizontal line. Furthermore, the fluctuations of the coincidences around the average value $n_{a v}=4.58$ that are clearly visible in fig 5 are fully consistent with the usual Poisson noise. This is evident in fig 6 where the relative frequency distribution of coincidences during a sidereal day is shown together with the Poisson distribution (full line) that corresponds to $n_{a v}=4.58$. We emphasize that no free parameter is used to draw the full line in fig 6 .

According to Eberhard, the model in [15] is only one of the possible realistic local models based on signals propagating at a superluminal velocity that can be proposed to justify the observed quantum correlations in EPR experiments. In particular, in such a model, the correlation between entangled photons is entirely due to the quantum communication and, thus, no correlation exists if they have not sufficient time to communicate. However, more complex models that are a combination of hidden variable theories and quantum communications could be proposed [16]. According to these models, the quantum correlation between entangled photons could be due in part to some correlation that is already present at the beginning when entangled particles are created (hidden variables) and in part to superluminal communications between them. In such a case, some residual correlation of entangled photons would be present also if they have not sufficient time to communicate and, thus, the number of coincidences that should be expected when there is no communication could be appreciably different from the value $n_{\max } / 2$ that characterizes totally uncorrelated events $\left[P_{12}\left(\gamma_{1}, \gamma_{2}\right) \neq P_{1}\left(\gamma_{1}\right) P_{2}\left(\gamma_{2}\right)=\right.$
1/4]. In such a case, one or more of the coincidences variations shown in fig 5 could be not due to the statistical noise but to the occurrence of some incomplete quantum correlation at a given time interval. Although this interpretation seems to be reasonably excluded by the satisfactory agreement between the experimental frequency distribution of the coincidences and the Poisson distribution (fig 6 , we decided to improve our analysis in order to evidence possible small systematic deviations. In order to reach this goal, we exploit an important feature of the Eberhard model: the lack of quantum correlation is expected to be a periodic phenomenon with a period of a sidereal day (in the ideal case $\rho=0$, the period becomes a half of a sidereal day). Then, a small systematic signal occurring at a given time of a sidereal day could be enhanced with respect to the statistical noise by summing the counts occurring at the same instant of $N$ different sidereal days. This procedure reduces the statistical noise by a factor $\sqrt{1 / N}$ but does not affect a possible periodic signal. For this reason we have repeated measurements over 21 sidereal days. The counts corresponding to the same time intervals of different sidereal days have been mediated. The results of this procedure are shown in fig 7 . Now, the statistical noise is appreciably reduced and the difference between the experimental counts and those expected for no correlated events (full horizontal line) is much more evident. Furthermore, no peak comes out from the statistical noise. In particular, the maximum departure of $n_{\text {coinc }}$ from the average value $n_{a v}=4.62$ is $\Delta n=1.95$ (the standard deviation in fig 7 is $\sigma=0.48)$. Such a variation corresponds to about $1 / 12$ of the expected variation $\Delta n=23$ for totally uncorrelated results.

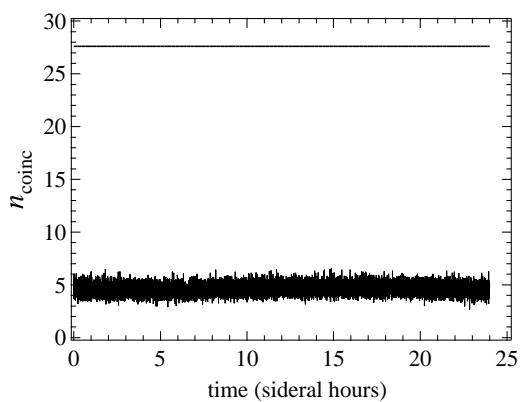

Figure 7: Number of coincidences with the acquisition time $\Delta t=4 \mathrm{~s}$ averaged over 21 sidereal days versus time.

Substituting our experimental parameters $\bar{\rho}=$ 
$1.6 \cdot 10^{-4}$ and $\Delta t=4 \mathrm{~s}$ in eq. (12) we can calculate the lower bound $\beta_{t, \text { min }}$ versus $\beta$ and $\chi$. Fig 8

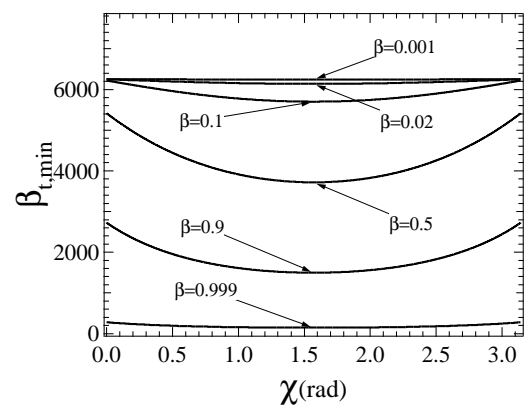

Figure 8: $\beta_{t, \min }$ versus $\chi$ for some values of $\beta$ obtained using eq. (12) with the experimental parameters $\bar{\rho}=1.6 \cdot 10^{-4}$ and $\Delta t=4 s$.

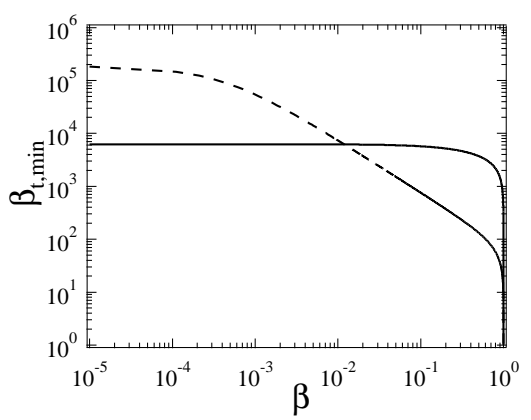

Figure 9: $\beta_{t, \min }$ versus $\beta$ for $\chi=\pi / 2$. The full line is obtained for $\bar{\rho}=1.6 \cdot 10^{-4}$ and $\Delta t=4 s$ using eq. 12 whilst the broken line corresponds to the parameters of the experiment in ref. 20] $\left(\bar{\rho}=5.4 \cdot 10^{-6}\right.$ and $\left.\Delta t=360 \mathrm{~s}\right)$.

shows the dependence of $\beta_{t, \text { min }}$ on $\chi$ for some values of $\beta$. Note that $\beta_{t, \min }$ is virtually independent of $\chi$ for $\beta \lesssim 10^{-2}$ and that, for a fixed $\beta$, the minimum value of $\beta_{t, \text { min }}$ is reached for $\chi=\pi / 2$. The relatively smooth dependence of $\beta_{t, \min }$ on $\chi$ is the direct consequence of two main features of our experiment: a) the choice of a East-West orientation of the axis connecting the polarizers; b) the choice of a relatively small $\Delta t[\sin (\Delta t / 2) \lesssim \bar{\rho}$ in eq.(12)]. In particular, if the detection axis makes a finite angle $\alpha$ with the East-West direction, a sharp decrease of $\beta_{t, \text { min }}$ occurs for $0 \leq \chi \lesssim \alpha$ and for $\pi-\alpha \lesssim \chi \leq \pi$ (see fig. 5 a of [20]). Fig.9] shows the dependence of $\beta_{t, \text { min }}$ on $\beta$ for $\chi=\pi / 2$. The full line corresponds to $\beta_{t, \text { min }}$ as obtained using the parameters $\bar{\rho}=1.6 \cdot 10^{-4}$ and $\Delta t=4 s$ of our experiment whilst the broken line is obtained using the parameters $\bar{\rho}=5.4 \cdot 10^{-6}$ and $\Delta t=360 \mathrm{~s}$ of ref. [20]. Note that our experimental values of $\beta_{t, \min }$ are virtually independent of $\beta$ up to $\beta \approx 0.1\left(\beta_{t, \min } \gtrsim 0.6 \cdot 10^{4}\right.$ for $\left.\beta<0.1\right)$. This is another direct consequence of relatively small value of $\Delta t$. The experimental results of Salart et al. [20] (broken line in fig. 9) show a much greater dependence on $\beta$ since $\sin (\Delta t / 2) \gg \bar{\rho}$ in their experiment. Note that the values of $\beta_{t, \text { min }}$ of the broken curve are more than one order of magnitude higher than those of the full curve if $\beta \lesssim 10^{-3}$ and become appreciably smaller for $\beta \gtrsim 10^{-2}$. We remind here that the velocity of the cosmic microwave background radiation reference frame is $v \approx 1.2 \cdot 10^{-3} c$ [19]. Finally, we emphasize that, for any value of $\Delta t$ and $\bar{\rho}, \beta_{t, \min } \rightarrow 1$ for $\beta \rightarrow 1$.

In conclusion, in this Letter we have experimentally investigated the possibility that the quantum correlation that characterizes EPR experiments can be due to some superluminal signal propagating in a preferred frame. Due to the choice of a West-East alignment of the polarizers and to the choice of a sufficiently small acquisition time, our experimental results are poorly dependent on both the modulus and the orientation of the velocity of the preferred frame. No evidence for the presence of superluminal signals has been observed in our experiment. Our experimental results provide a lower bound for the velocity of possible superluminal signals as shown in the full curve of fig 9

\section{Acknowledgments}

The authors greatly acknowledge Marco Bianucci for valuable technical support, Giuseppe Cocciaro for the support given at the beginning of the experiment and Nicolas Gisin for critical comments.

\section{References}

[1] A. Einstein, B. Podolsky and N. Rosen, Phys. Rev. 47 (1935) 777.

[2] J. S. Bell, Physics 1 (1964) 195.

[3] J. F. Clauser, M. A. Horne, A. Shimony and R. A. Holt, Phys. Rev. Lett. 23 (1969) 880.

[4] J. F. Clauser and M. A. Horne, Phys. Rev. D 10 (1974) 526.

[5] S. J. Freedman and J. F. Clauser, Phys. Rev. Lett. 28 (1972) 938.

[6] A. Aspect, J. Dalibard and G. Roger, Phys. Rev. Lett. 49 (1982) 1804.

[7] G. Weihs, T. Jennewein, C. Simon, H. Weinfurter and A. Zeilinger, Phys. Rev. Lett. 81 (1998) 5039.

[8] M. A. Rowe, D. Kielpinski, V. Meyer, C. A. Sackett, W. M. Itano, C. Monroe, D. J. Wineland, Nature 409 (2001) 791.

[9] J. W. Pan, D. Bouwmeester, M. Daniell, H. Weinfurter and A. Zeilinger, Nature 403 (2000) 515. 
[10] A. Zeilinger, Phys. Lett. A 118 (1986) 1.

[11] A. Aspect, Nature 398 (1999) 189.

[12] P. Grangier, Nature 409 (2001) 774.

[13] W. Tittel, J. Brendel, H. Zbinden and N. Gisin, Phys. Rev. Lett. 81 (1998), 3563;

[14] M. Genovese, Phys. Rep. 413 (2005) 319.

[15] P. H. Eberhard, A realistic model for Quantum Theory with a locality property, in W. Shommers (Ed.), Quantum Theory and Pictures of Reality, Springer, Berlin, 1989 and, also, P. H. Eberhard, Lawrence Berkeley Lab., LBL-34575, Aug. 1993, http://www.osti.gov/bridge/purl.cover.jsp?purl=/10191801-vaC5R6/

[16] D. Bohm and B. J. Hiley, The Undivided Universe, Routledge, (1993)

[17] see, for instance, C. Möller, The theory of relativity, Clarendon Press, Oxford (1952), pagg. 52-53.

[18] S. Liberati, S. Sonego and M. Visser, Ann. Phys. 298 (2002) 167

[19] V. Scarani, W. Tittel, H. Zbinden and N. Gisin, Phys. Lett. A 276 (2000) 1.

[20] D. Salart, A. Baas, C. Branciard, N. Gisin and H. Zbinden, Nature 454 (2008) 861.

[21] B. Cocciaro, arXiv:quant-ph/0512202v1

[22] P. G. Kwiat, E. Waks, A. G. White, I. Appelbaum and P. H. Eberhard, Phys. Rev. A 60, R773 (1999).

[23] J. B. Altepeter, E. R. Jeffrey and P. Kwiat, Optics Express 13 (2005) 8951 and also G. M. Akselrod, J. B. Altapeter, E. R. Jeffrey and P. G. Kwiat, Optics Express 15 (2007) 5260. 